\title{
Autotuning of Parallel Cascade Control using Setpoint Relay
}

\author{
M.Bharathi \\ Dept. of EIE, \\ Bharath \\ University, \\ Chennai. \\ 600073, India.
}

\author{
S.Renganathan \\ Rrase College of \\ Engineering, \\ Padappai 601301, \\ India.
}

\author{
C.Selvakumar \\ Dept.of EIE, \\ St.Joseph's \\ College of \\ Engineering, Anna \\ University, \\ Chennai-600 119, \\ India.
}

\author{
V.Vijayan \\ Dept.of EIE, \\ St.Joseph's \\ College of \\ Engineering, \\ Anna University, \\ Chennai-600 119, \\ India.
}

\author{
R.C.Panda \\ Dept.of Chemical \\ Engineering, \\ CLRI, Adayar, \\ Chennai-600020, \\ India.
}

\begin{abstract}
A new approach to tune the PI controller for parallel cascade control using setpoint relay autotuning method is proposed in this paper. The setpoint relay autotuning is found to be effective as that of the relay feedback method without switching off existing controller. The proposed method is found to be more advantageous for finding out ultimate gain and ultimate period and tested in computer simulation using various tuning methods. The results show that reasonable control performance can be achieved using IMC-PID method.
\end{abstract}

Keywords: Parallel cascade, PID controller, set point relay, Autotuning.

\section{INTRODUCTION}

In process industries, cascade system is desirable to reduce the effects of possible disturbances and to improve the dynamic performance of the closed loop system. In the traditional series cascade control, both the manipulated variable and disturbance variables affect the primary output through the intermediate (secondary) output. The parallel cascade control was first proposed by Luyben (1973). The overhead composition control of a distillation column, cascaded onto the control of tray temperature, is a typical example of a parallel cascade control system. The reflux flow rate (manipulated variable) and the feed flow or composition (disturbance, d) affect, both, the purity of the overhead product (primary output, y1) and the tray temperature (secondary output, y2). The control objective is to maintain the overhead composition at the setpoint. The output of the composition controller resets the setpoint for the temperature controller. By controlling the tray temperature in the cascade manner, the variation in the feed can be compensated before it disturbs the product composition. In general, parallel cascade control is appropriate when the secondary loop has a faster dynamic response and the rejection of the disturbance in the secondary output reduces the steady state output error in the primary loop. The parallel cascade control is also beneficial when measurements of the primary output are sampled infrequently and/or with long time delays. In general, parallel cascade control is very much appropriate when the secondary loop has a faster dynamic response and the rejection of the disturbance in the secondary output reduces the steady state output error in the primary loop. The parallel cascade control is also beneficial when measurements of the primary output are sampled infrequently and/or with long time delays. In series cascade control the secondary process is not concerned since it is used for disturbance rejection alone where as in parallel the secondary process has major role contributing in disturbance rejection and to improve the dynamic performance of the closed loop system.

In spite of clear benefits of the parallel cascade control and its wide-spread use in process industries, the design on the parallel cascade control systems has attracted relatively little research. Yu (1988) proposed an efficient interaction measure to determine whether the parallel cascade control is advantageous or detrimental to load response Rao et.al.,(2009) incorporated a delay compensator in the primary loop of the parallel cascade control system and the secondary controller is designed using the internal model control (IMC) method. Martin et al.,(1996) demonstrated the superior performance and failure tolerance that can be achieved with the parallel control strategy compared to cascade control and single-input, single-output control techniques. Junghui et al., (2005) evaluated the parallel cascade control system scheme against an achievable performance standard based on the minimum variance and the Diophantine decomposition for the parallel cascade control system. Uma and Rao (2009) designed the primary controller using direct synthesis method in parallel cascade controllers in the chemical and bioprocess to improve the dynamic performance of a control system in the presence of disturbances. Karthikeyan et.al., (2010) designed a fuzzy based control for the primary controller and IMC for secondary controller to improve the performance of the parallel cascade control. Raghavan and Radhakrishnan (2010) used the composition from the conventional composition analyzer as the primary variable and considered tray temperature as the controller output, which in turn is taken as the set point by the secondary controller and manipulates vapour and reflux flow rates to control the composition in parallel cascade control scheme.

\section{PARALLEL CASCADE CONTROL}

A parallel cascade system is one in which both the manipulated variable and the disturbance affect the primary and the secondary output through the parallel actions while in a series cascade both actions on the primary output take place through secondary one. It is clear that the series or parallel nature of a cascade is related to the characteristics of the process under analysis and not to the control system to be designed.

Analysis and design of conventional controllers for parallel cascade control structures has been the subject of a work by $\mathrm{Yu}$ (1998). The parallel cascade structure proposed by Luyben (1973) is shown in Figure 1. It is apparent that both the manipulated variable and the disturbance affect the primary and secondary outputs through parallel transfer functions. 
Luyben (1973) in his paper studied the difference between series and parallel and found that the control is based on the process and not on the structure.

\subsection{Series Equivalent of Parallel Cascade Structure}

The parallel cascade scheme is compared initially to the series cascade one in order to show differences and similarities; this comparison is useful to understand how to extend control structures which are effective for the series cascade case to the parallel cascade. Semino and Brambilla (1996) used block diagram transformation rules to turn the structure into one that resembles the series cascade as shown in Figure 2.

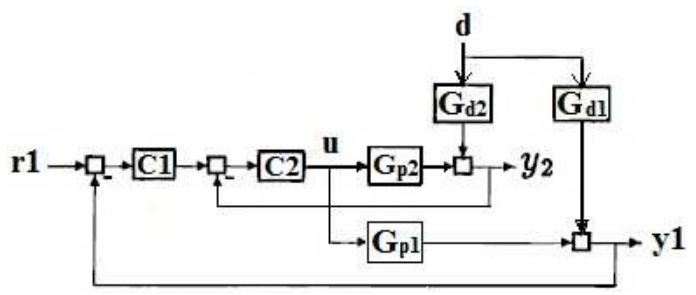

Figure 1 Parallel cascade structure

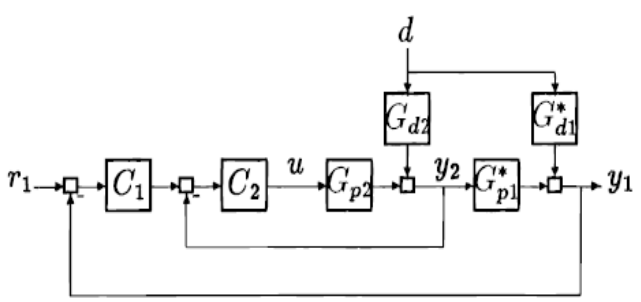

Figure 2 Series Equivalent of Parallel cascade

\section{structure}

2.1.1 Comparison of Parallel Cascade and Series Equivalent of Parallel Cascade

It is difficult to tune the secondary controller when we take the parallel cascade structure. This can be made easy by using series equivalent of parallel cascade structure. The responses are shown in Figure 3. Here the tuning is done by using Zeigler-Nichols tuning method. The response of the series equivalent is almost comparable to the parallel structure by implementing autotuning (Vivek and Cidambaram, 1997).

\subsubsection{Autotuning of Parallel Cascade Control}

Despite the development of more advanced control strategies, the majority of industrial control systems still use PID controllers because they are standard industrial components, and their principle is well understood by engineers. However it is common that many controllers are poorly tuned. If disturbance or if the process dynamic characteristics is large, the tuning of a PID controller is often difficult and the need for autotuning arises.

Recently, many techniques have been proposed for the autotuning of PID controllers, viz. relay feedback (Hang et al.,2002).Amongst the most popular autotuning approach in industry and academic research is perhaps relay based autotuning. A similar scheme based on setpoint relay has been proposed by Schei (1992), where the model identification and control design are performed via on-line iteration.

\subsubsection{Setpoint Relay Autotuing}

In this paper the setpoint relay autotuning projected by Luo et al (1998) for parallel cascade structure is analogous to that of a relay feedback experiment. The control parameters are determined from the knowledge of critical gain and critical period. In relay feedback, the existing controller is replaced by a relay so the processes will oscillate in a limit cycle. The critical gain and critical period are obtained from the amplitude and the frequency of this oscillation. In setpoint relay, there is no need to switch off the existing controller. The system is excited by connecting a relay and an extra feedback signal to the setpoint, as shown in Figure 4. The relay output is connected to the setpoint of existing PID controller.

\section{SIMULATION STUDY}

To evaluate the proposed method, computer simulations were performed for the process projected by Luyben (1973) with The primary and secondary transfer functions are used in series equivalent of parallel cascade control.

$$
\begin{aligned}
& p_{1}=p_{d 1}=\frac{e^{-4 s}}{(20 s+1)} \\
& p_{2}=p_{d 2}=\frac{1}{(10 s+1)}
\end{aligned}
$$

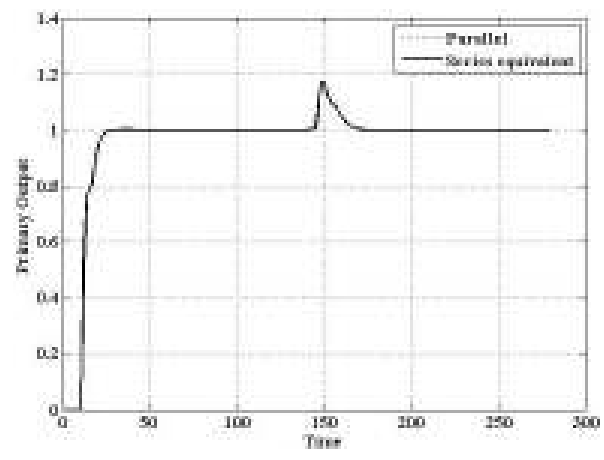

Figure 3. Primary output response of Parallel and

$$
\text { series equivalent structure }
$$

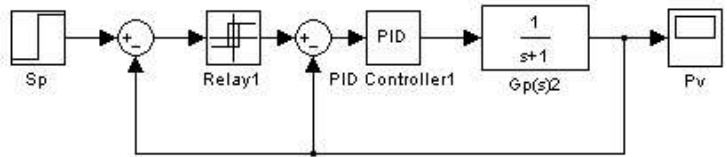

Figure 4. Setpoint Relay Autotuning

The block diagram for this process is shown in Figures

7, 8. Autotuning is done using setpoint relay method. The 
proposed method is used to find the ultimate gain and ultimate period. Using some tuning rules (O'Dwyer, 2003) listed in Tables 1,2 the $K_{p}$ and $T_{i}$ values are determined for the controller.

Figure 5. represents the closed loop system performance of the parallel cascade control system with the proposed method using various controller tuning techniques for a load change. Performance evaluation IAE, ISE, ITAE of the closed loop schemes shows that IMC-PID is better as listed in the Table 3.

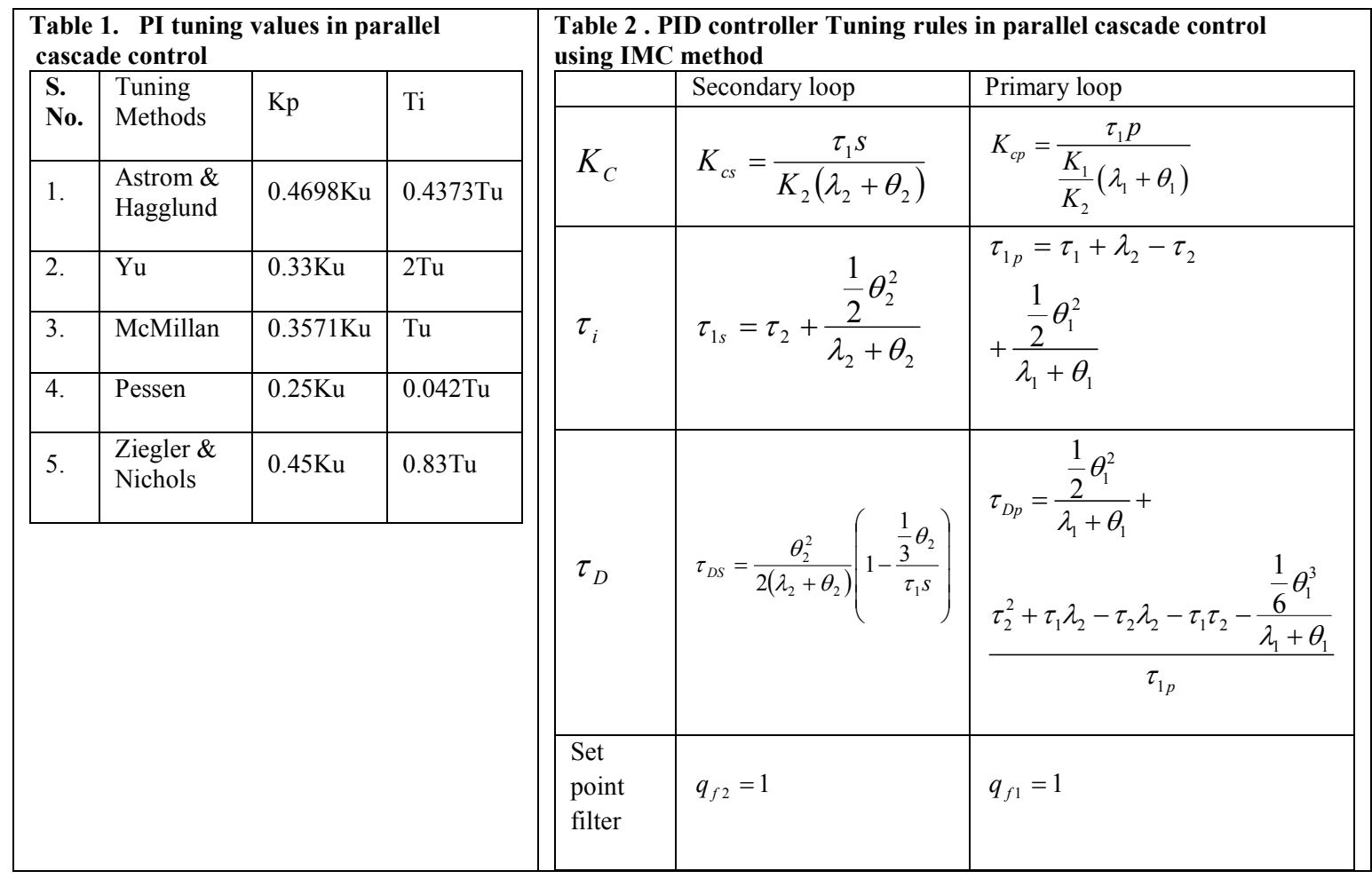

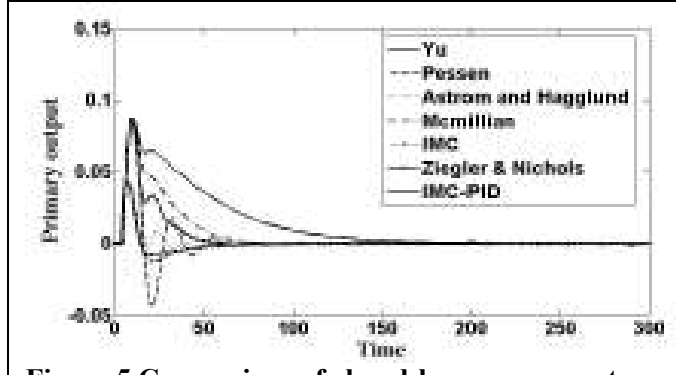

Figure 5.Comparison of closed-loop responses to a load change

Table 3. Comparison response for various tuning techniques to a load change

\begin{tabular}{|l|l|l|l|l|}
\hline S.N & Tuning & \multicolumn{4}{|l|}{ Performance criteria } \\
\cline { 3 - 5 } o & methods & ISE & IAE & ITAE \\
\hline 1. & $\begin{array}{l}\text { Astrom \& } \\
\text { Hagglund }\end{array}$ & 0.3641 & 0.7479 & 10.15 \\
\hline 2. & Yu & 0.1858 & 3.843 & 158.8 \\
\hline 3. & McMillan & 0.09191 & 1.865 & 42.52 \\
\hline 4. & Pessen & 0.03948 & 0.7177 & 8.453 \\
\hline
\end{tabular}

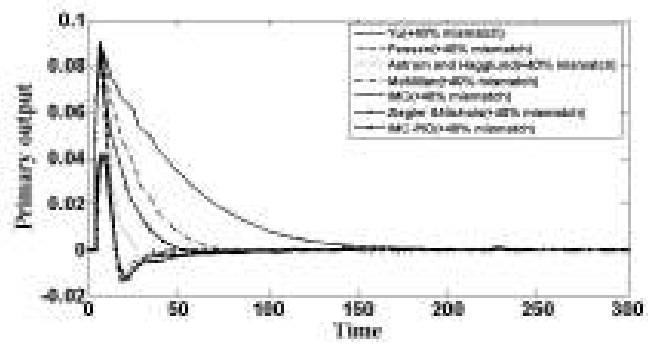

Figure 6. Comparison of closed-loop responses to a load change under process model uncertainty

Table 4. Response for various tuning techniques to load change under process model uncertainty

\begin{tabular}{|c|c|c|c|c|}
\hline S.No & Tuning & \multicolumn{4}{|l|}{ Performance criteria } \\
\cline { 3 - 5 } & methods & ISE & IAE & ITAE \\
\hline 1. & $\begin{array}{c}\text { Astrom \& } \\
\text { Hagglund }\end{array}$ & 0.3001 & 2.725 & 49.94 \\
\hline 2. & $\mathrm{Yu}$ & 0.8581 & 7.78 & 306.8 \\
\hline 3. & McMillan & 0.4566 & 3.624 & 77.13 \\
\hline 4. & Pessen & 1.834 & 16.84 & 1369 \\
\hline
\end{tabular}




\begin{tabular}{|c|c|c|c|c|c|c|c|c|c|}
\hline 5. & $\begin{array}{l}\text { Ziegler \& } \\
\text { Nichols }\end{array}$ & 0.05869 & 1.259 & 23.13 & 5. & $\begin{array}{c}\text { Ziegler \& } \\
\text { Nichols }\end{array}$ & 0.05537 & 1.125 & 33.01 \\
\hline 6. & IMC & 0.0237 & 0.9942 & 27.03 & 6. & IMC & 0.0237 & 0.9942 & 27.03 \\
\hline 7. & IMC-PID & 0.01076 & 0.5817 & 8.36 & 7. & IMC-PID & 0.01076 & 0.5817 & 18.36 \\
\hline
\end{tabular}

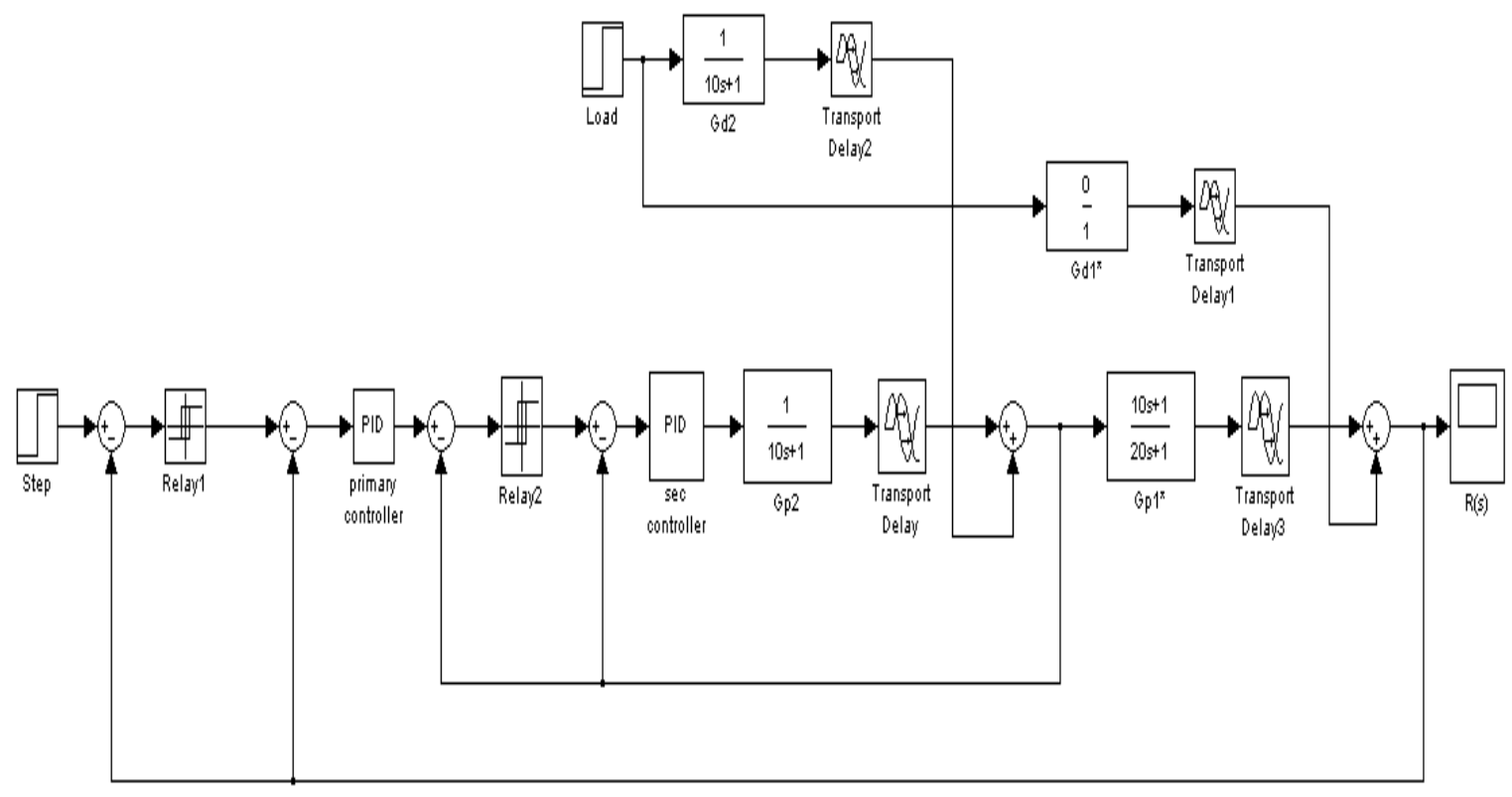

Figure 7. Implementation of setpoint relay to the series equivalent of parallel cascade structure

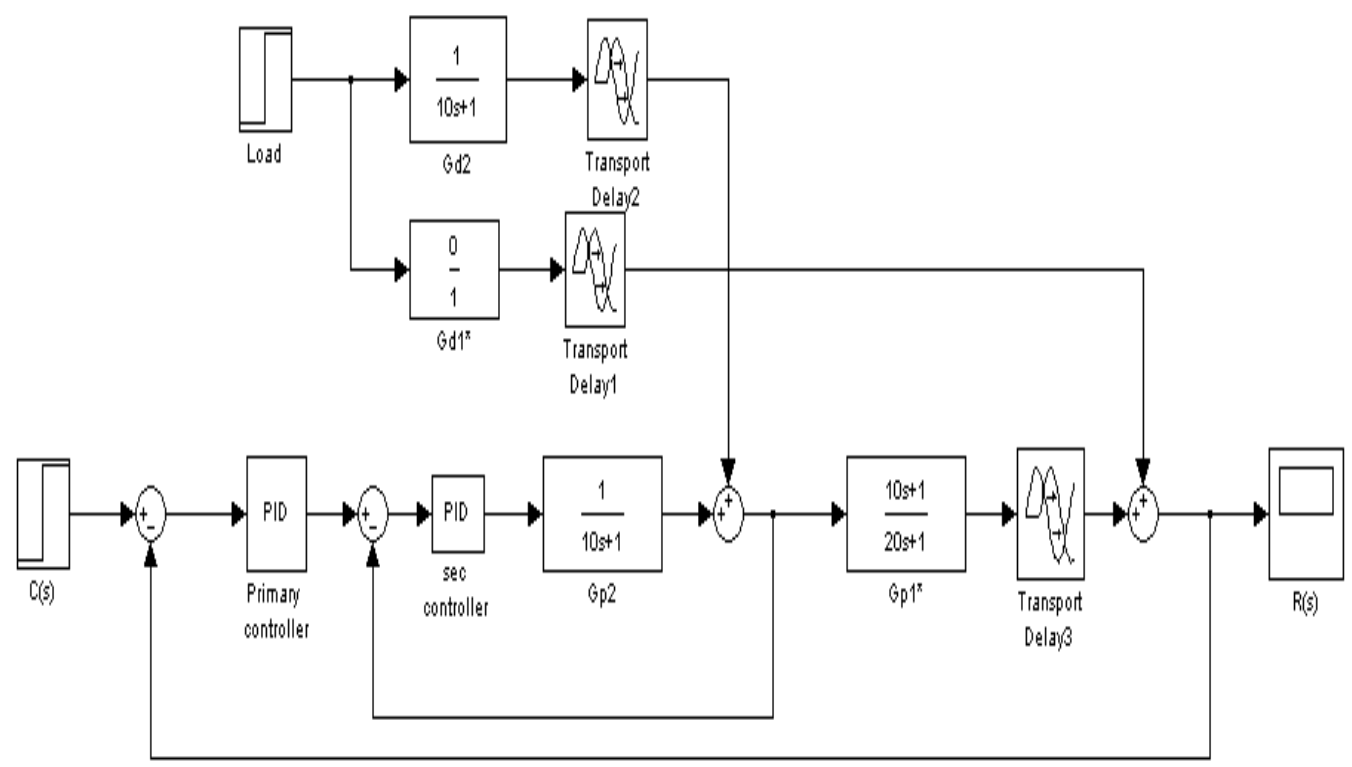

Figure 8. Implementation of PI controller to the series equivalent of parallel cascade structure 
To investigate the robustness of the proposed controller, we suppose that there exist $+40 \%$ errors for estimating process model parameters in both the primary and secondary loops. For instance, the process gain, the time constant and the time delay are actually $40 \%$ larger than those in the nominal model. The closed-loop response by the various controller tuning techniques with the proposed method is provided in Figure 6 and a comparison with performance criteria as given in Table 4. The performance of the IMC-PID holds the control system robust stability as well in the presence of the severe process uncertainty.

\section{CONCLUSION}

An efficient structure for parallel cascade control is proposed. A set of procedures for the autotuning of parallel cascade control structures is proposed and the results are compared. Performance evaluation of the closed loop responses with the setpoint relay autotuning is very effective and implemented in tuning the series equivalent of parallel cascade structure. The IMC-PID with the proposed method provides better result.

\section{REFERENCES}

[1]. Hang.C, Astrom.K, and Wang.Q 2002., "Relay feedback autotuning of process controllers - a tutorial review", Journal of Process Control, vol. 12, no. 1, pp. 143-162.

[2]. Junghui Chen, Shun-Chi Huang and Yuezhi Yea 2005, "Achievable Performance Assessment and Design for Parallel Cascade Control Systems, Journal of Chemical Engineering of Japan, Vol. 38,No. 3, pp.188-201.

[3]. Karthikeyan.R, Tapio Pahikkala et.al. 2010, Fuzzy Logic Based Control for Parallel Cascade Control, ICGSTACSE Journal, Vol.10, Issue 1, pp. 39-48.

[4]. Luyben.W 1973. "Parallel cascade control", Ind. Eng. Chem. Fund. 12 (1973), pp 463-467.
[5]. Martin Pottmann, et.al.1996, "A parallel control Strategy abstracted from the baroreceptor reflex Original Research Article Chemical Engineering Science, Vol.51, Issue 6, pp. 931-945.

[6]. O'Dwyer.A, 2003, "Handbook of PI and PID Controller Tuning Rules", London:Imperial College Press.

[7]. Raghavan S R V, Radhakrishnan T K. 2010, "Parallel Cascade Control System Design for Ideal Reactive Distillation Column", IE(I) Journal-CH, Vol. 90, 27,pp22-27.

[8]. Rao Seshagiri.A et.al.2009, "Enhancing the performance of parallel cascade control using Smith predictor", ISA Transactions, Vol.48, Issue 2, pp. 220-227.

[9]. Rongfu Luo S. Joe Qin et.al. 1998, “A New Approach to Closed-Loop Autotuning for Proportional-Integral-Derivative Controllers", Ind. Eng. Chem. Res., vol.37 (6), pp 2462-2468.

[10]. Schei Tor Steinar 1992, "A method for closed loop automatic tuning of PID controllers", Automatica, Vol.28, Issue 3, pp.587-591

[11].Semino D., Brambilla A.1996, "An efficient structure for parallel cascade control", Ind. Eng. Chem. Res. Vol.35, pp.1845-1852.

[12].Vivek.S,Chidambara.M. 2007, "Relay Autotuning of Parallel Cascade control", Proceedings of the World Congress on Engineering and Computer Science,WCECS 2007, October 24-26, 2007.

[13].Uma S. and Seshagiri Rao A.2009, "Parallel cascade control strategy for unstable continuous bioreactor", Theories and Applications of chemi. Eng., vol.15, no.2, pp1375-1382.

[14].Yu.C.C 1998, "Design of parallel cascade control for disturbance rejection", AIChE J, vol. 44, pp.1833-1838. 